\title{
Does gender influence the outcome of ischemic heart disease?
}

\author{
Michał Tomaszewski ${ }^{1}$, Weronika Topyła ${ }^{1}$, Bartosz Grzegorz Kijewski ${ }^{1}$, Paweł Miotła ${ }^{2}$, Piotr Waciński ${ }^{1}$ \\ ${ }^{1}$ Department of Cardiology, Medical University of Lublin, Poland \\ ${ }^{2} 2^{\text {nd }}$ Department of Gynaecology, Medical University of Lublin, Poland
}

\begin{abstract}
Diseases of the cardiovascular system (myocardial infarction, stroke, heart failure, hypertensive heart disease, cardiomyopathy) account for $40 \%$ of all deaths in men and up to $49 \%$ of all deaths in women. For a long time it was thought that the clinical picture of ischemic heart disease in men and women was similar. Now, however, there are more reports suggesting that diverse manifestations of the symptoms of ischemic disease may be related to differences between sexes.

The disparity between women and men is also evident in the diagnostic process, and various pathological mechanisms of cardiovascular diseases, in particular myocardial ischemia in men and women, affect the differences in the results of diagnostic tests. Vasomotor dysfunction is particularly frequent in women, as their coronary vessels are more sensitive to the catecholamines released during mental stress, resulting in spasm and ischemic myocardium. Moreover, a much lower dose of acetylcholine induced vasoconstriction, which indicates that women are more sensitive to this neurotransmitter. Therefore, coronary vasomotor disorders in the form of epicardial and microvascular dysfunction are more often seen in women. All these mentioned factors resulted in higher mortality and poorer quality of life of women suffering from ischemic heart disease.
\end{abstract}

Key words: ischemic heart disease, myocardial ischemia, gender differences, women.

\section{Introduction}

Cardiovascular diseases are still the leading cause of death, morbidity and disability, not only in Europe but around the world. Of non-communicable diseases leading to mortality, these form the largest percentages of cases for persons under the age of 70 years [1]. Indeed, diseases of the cardiovascular system (myocardial infarction, stroke, heart failure) account for $40 \%$ of all deaths in men and up to $49 \%$ of all deaths in women. For comparison, lung cancer accounts for $6 \%$ of this amount in men, while breast cancer causes death in $2 \%$ of all women [2]. In addition to the domestic and social consequences, cardiovascular disease also has an effect on a country's economy by significantly reducing the number of people of productive age. Importantly, cardiovascular diseases are not only currently the most common cause of death of women in Europe and the United States, but a constant increase in their frequency is seen. This is only partially explained by an aging population.

In recent years, more attention has been paid to assessing the impact of gender on the development of these diseases. Specifically, the aforementioned increase of morbidity and mortality from diseases of the cardiovascular system was noted in women, resulting in quality of life issues through risk factors for cardiovascular disease. Hence, knowledge of the multidimensional effects of hypertension and other risk factors for cardiovascular disease is essential in reducing the real threat of cardiovascular disease in women, and thus improving their quality of life.

Particular attention should be paid to ischemic heart disease, as this dominates among cardiovascular deaths. In women, detection and evaluation of coronary heart disease is still a challenge, and early detection of the risk occurrence is very important. For example, women with suspected angina wherein pathological changes in coronarography were not detected often experience chest pain and have a higher probability of acute coronary syndrome than men [3]. Most recent reports highlight the significant role of sex in the incidence, course and mortality especially in the case of acute coronary syndromes. With regard to these, they result in a greater mortality in women compared to men. This difference is notably significant for patients under 75 years of age, as above that age, mortality from acute coronary syndrome is comparable $[4,5]$. Sabbag et al. noted that the difference in mortality is particu- 
larly evident in patients less than 55 years old, wherein among females, researchers saw a greater proportion of in-hospital mortality, increased 30-day and 5-year mortality rate and a higher incidence of cardiac and cerebral complications within 30 days of the cardiac incident [6]. Other studies confirm the above-mentioned relationships, and through data mining, a larger 30-day mortality figure is seen for women under 60 years of age after ST elevation myocardial infarction (STEMI). Here, in terms of data capture, the forefront word string was "female", then "diabetes mellitus", "history of PCl", "heart rate" and Killip class of 2 or higher [7].

\section{The role of risk factors}

In recognizing the importance of this issue, especially in women, it is crucial to pay attention to the leading risk factors for cardiovascular disease which directly increase the incidence of cardiovascular complications, stroke and myocardial infarction. These are hypertension, dyslipidemia and obesity. Among patients who experienced an acute cardiac event, women more often than men had diabetes (34\% vs. $24 \%$ ) and hypertension (47\% vs. $37 \%$ ) [3]. In addition, women were obese more often ( $25.5 \%$ vs. $18.5 \%)$, yet were less likely to be smokers [8], although, recently, the number of young women who smoke has rapidly increased.

With regard to women, smoking increases cardiovascular risk to a greater extent than in men. In addition to other harmful activities, smoking a single cigarette causes an increase of blood pressure of $5-10 \mathrm{~mm} \mathrm{Hg}$ for about 30 minutes. Furthermore, smoking only 1-4 cigarettes a day significantly increases a woman's risk of a heart attack, and smoking a greater number of cigarettes can accelerate the occurrence of acute coronary syndrome even 20 years after having quit smoking [9].

There are several states and factors in the development of coronary heart disease in women that are unique, such as early menopause or menarche, and eclampsia, preeclampsia during pregnancy, as well as systemic inflammatory disorders [10, 11]. Moreover, as estrogens have a protective effect on the cardiovascular system, such a situation makes women who suffer from coronary heart disease show the effects about 10 years later than do men [12]. Therefore, postmenopausal women are considered to be at high risk of cardiovascular disease. Further, a reduction in ovarian function and a reduction in the level of estrogens during menopause induce accelerated aging of the vascular system. The effects include endothelial dysfunction and stiffening of the walls of the large arteries via an unspecified mechanism. Hence, there is a higher incidence of cardiovascular disease in women who have experienced premature menopause, independent of other risk factors such as hypertension, obesity and smoking $[13,14]$.

\section{The course of ischemic heart disease}

For a long time it was thought that the clinical picture of ischemic heart disease in men and women was similar. Now, however, there are more reports suggesting that diverse manifestations of the symptoms of ischemic disease may be related to differences between the sexes. Thus, this should be considered when assessing patients with chest pain. It should be remembered that the specificity of the symptoms of angina in women may hinder/hamper making a proper diagnosis. In a study of 532 patients who had experienced an ST-elevation myocardial infarction, it was found that women are far less likely than men to experience chest pain (74\% vs. 93\%) during the incident. In addition, women are less likely to interpret the symptoms as being of heart origin. Hence, it has been concluded that being of female gender is the strongest predictor of non-chest presentation [15].

This study also concluded that there was no difference between the sexes in the character of pain, but in women, pain radiating to the arm, neck and back is recorded twice as often as in men. In addition, women more often feel nausea, and fear often accompanies the above-mentioned symptoms [15]. Other studies have found that women more frequently experience atypical chest pain and receive higher Killip class scores $(>1)$. Furthermore, women develop cardiogenic shock more commonly during hospitalization than do men. They are also rated higher in the GRACE score (>140) [3].

There is a greater difference between the sexes in younger patients in the presentation of symptoms of myocardial ischemia. Women rarely present the classic symptoms (31\% as compared with $42 \%$ in men), but more often feel weakness, palpitations, nausea, pain radiating to the jaw, shoulder or back and loss of appetite [16, 17]. Moreover, women rarely present symptoms within the first two hours of the start of ischemia, especially in patients under 60 years of age [7]. In connection with these atypical symptoms, female patients seek emergency medical help later than do men, have higher risk profiles during the admission, and have longer total ischemic times (414 min vs. $328 \mathrm{~min}$ ) [3]. Additionally, on hospital admission, a greater percentage of women are haemodynamically unstable and frequently exhibit a ventricular systolic dysfunction (32.9\% vs. 24.9\%) [8]. Such a situation leads to reduced recognition of myocardial ischemia in women.

\section{Diagnostic process}

Diagnosing patients with chest pain is a challenge because of the diversity of the clinical picture of angina in women, as well as the different values generated through non-invasive diagnostic tests. Ascertaining the 
optimal method of treatment in women with suspected or known coronary artery disease has been the subject of numerous discussions. Herein, the restricted use or incorrect interpretation of non-invasive tests has led to the application of improper therapy and has enhanced the incidences of life-threatening cardiac events and ischemic complications in women $[18,19]$.

Recent studies have shown that mortality after effectively performed percutaneous coronary intervention $(\mathrm{PCI})$ in the case of STEMI is comparable between sexes [20]. In contrast, it was noted that women were treated significantly less invasively and were directed more often towards pharmacological treatment, as coronary stenting and bypass surgery were more frequently applied in treating men as compared to women [20]. Additionally, in the study of Sadowski et al., of a group of patients with STEMI, only $35.8 \%$ of all woman underwent primary $\mathrm{PCl}$ earlier than 12 hours from symptom onset, as compared with $44 \%$ of all men [21].

In a higher proportion of women with chest pain, negative coronary arteriography is observed. Probably, the reason for this is the presence of non-obstructive atherosclerosis. This latter serves as a pathophysiological mechanism of ischemic heart disease (IHD) [22]. Recent reports show that women are less likely to undergo cardiac rehabilitation after an acute cardiac incident. This situation suggests a disturbing difference in the quality of medical care between genders. Indeed, differences are noticeable at every stage of the process of cardiac rehabilitation: from qualification, to performing the procedures. All this leads to the poorer quality of life and increased mortality in women, as compared to men, after acute cardiac incidents [23].

The differences in results of diagnostic tests impact upon this. This distinction is particularly evident in the case of a diagnostic stress test. This is one of the basic studies in symptomatic women with normal ECG and moderate risk of coronary disease. Herein, women express a lower sensitivity and specificity in the detection of coronary artery disease (CAD) as compared to men [24]. In view of the fact that, in women, the basis of the symptoms of ischemic disease is often vascular remodeling and non-obstructive plaque, the results of applied tests should be interpreted differently. The traditional approach, defining a positive cardiac stress test, when it comes to a reversible ST segment depression of at least $1 \mathrm{~mm}$, may lead to under-diagnosis in women [25]. Therefore, women with a normal stress test and abnormal ECG results should be regarded as being true positives for CAD.

In case of doubt as to the correctness of the stress test, the alternative is to perform stress echocardiography, as in such a test, the sensitivity and specificity are the same for both sexes [26]. In CAD diagnosis, single proton emission computed tomography (SPECT) and positive emission tomography (PET) are effective diagnostic tools especially with regard in patients with moderate cardiovascular risk. Herein, PET and SPECT demonstrate sufficient diagnostic accuracy in the evaluation of clinically significant coronary artery disease in women [27].

Cardiovascular magnetic resonance (CMR) and multi-slice-computed tomography (MSCT) also show high predictive values in CAD diagnosis or in assessing the potential risk of cardiac events, and are especially valuable in patients with moderate risk of coronary disease. This allows the assessment of morphological changes in the coronary arteries, of calcifications and the size and severity of the stenosis. Moreover, they are useful in quantitative assessment of coronary flow and coronary reserve. CMR also allows for assessment of functional ischemic hypo-perfusion through pharmacological stimulation. It is important to note that both of these diagnostic tools retain the same sensitivity and specificity for both sexes [28, 29].

Recently, MSCT has become more important in the diagnosis of ischemic heart disease. MSCT is particularly useful in the case of low and moderate risk of IHD because it allows for an exclusion of non-invasive atherosclerotic lesions in the coronary arteries (high negative predictive value), the evaluation of the degree of calcification and the potency of myocardial contractility.

The range of clinical applications of MSCT has expanded rapidly since its introduction due to the universality of this method (this also applies to magnetic resonance imaging), because it enables an overall evaluation of vascular and cardiac structures in one study.

An additional test, computed tomography angiography (CTA), allows the diagnostician to image the system of the coronary vessels, its lumen and the wall of vessels. Thus, it is widely used in the evaluation of the degree of coronary artery stenosis, of changes in the wall and vessel anomalies, in the structure of vascular malformations and myocardial bridging. In addition, MSCT gives the opportunity to evaluate the coronary calcium score index (CS), which is now considered as the primary method for defining the independent risk myocardial bridging factor for coronary events [30].

\section{Vascular and biological factors}

Approximately $60 \%$ of all women who experience the symptoms of angina have neither significant stenosis in the coronary arteries, nor current massive atherosclerotic plaques [31, 32]. The presence of angina without significant vasoconstriction occurs more frequently in women than in men, and is associated with an increased risk of cardiac events in these patients [33]. This often results in under-diagnosis of females for ischemic heart disease and hence less frequent application of appropriate therapy. The aforementioned fact is one of the reasons for the higher mortality in 
women with coronary heart disease compared to men. This also translates into a decreased quality of life in patients with such symptoms. For women, the most likely causes of these phenomena and of worse outcomes in the course of coronary heart disease are vascular biological factors. These include smaller diameter of coronary vessels, less collateral flow and increased arterial stiffness [34]. Furthermore, coronary vasomotor disorders in the form of epicardial and microvascular dysfunction are more often seen in women.

In people without significant coronary artery disease, future cardiovascular events are limited to those who experienced a decrease of coronary blood flow in response to the intra-coronary infusion of acetylcholine. Thus, endothelial dysfunction is an independent predictor of cardiovascular events. Existing temporary or stable microvascular dysfunction, in turn, may contribute to the development of acute coronary syndrome by reducing coronary flow. This situation enhances the endothelial dysfunction in epicardial coronary arteries and can lead to thrombus formation $[35,36]$. In the study of Aziz et al., in the acetylcholine spasm provocation test, vasomotor dysfunction was found significantly more often in women than in men (70.2\% vs. $43.1 \%)$. Furthermore, a significant difference was observed on being dosed with acetylcholine. This gives positive results in both sexes, but in women a much lower dose induced vasoconstriction. Hence, this indicates that women are more sensitive to acetylcholine [37].

These tests explain the phenomenon of absence of pathological changes in percutaneous coronary intervention $(\mathrm{PCl})$ in patients with symptoms of cardiac angina. This is a serious problem in the treatment of women with ischemic heart disease because it is one of the most important causes of under-diagnosis in this group of patients. Therefore, in these cases, the diagnostic process of cardiac stress test, stress echocardiography, or cardiovascular magnetic resonance must be expanded so as to allow a proper assessment of myocardial ischemia.

\section{Mental stress induced myocardial ischemia}

Vasomotor dysfunction of the coronary arteries and their greater susceptibility to constriction under the influence of catecholamines is also the basis for other adverse events associated with ischemic heart disease. As evidenced, microvascular spasm-induced myocardial stress can be a cause of chest pain in the course of CAD [38]. Indeed, a study of 950 patients showed that women with stable IHD (in contrast to acute coronary syndrome) more often than men experienced symptoms of angina. Moreover, in this group of patients, women were twice as susceptible to the development of ischemia under the influence of psychological stress. No such phenomenon was observed in the male popula- tion. Therefore, it is concluded that women with stable IHD are more prone to the symptoms of angina under stress [39]. This phenomenon is called mental stress induced myocardial ischemia (MSIMI) and draws attention to the role of psychological factors in the course of CAD. MSIMI may also be responsible for symptoms of angina in patients, and increased episodes of chest pain can be a sign of mental stress. Herein, angina symptoms are usually associated with feelings of anxiety, chronic stress or depression [40]. MSIMI has also been twice as often identified in young patients with recent myocardial infarction. In this group, women were also found to have a worse psychological and sociodemographic profile than men, including lower income, less education, greater suffering from depression and greater exposure to chronic stress. In addition, more women take antidepressants [41]. Moreover, IHD is considered as the leading cause of disability and death in women in the United States [42].

These studies indicate the role of mental stress in ischemic heart disease. It can be concluded that the above-mentioned dependences translate into a poorer quality of life of women with IHD. Therefore, it is important to take care of the psychological comfort of patients, especially women with symptoms of angina, and if necessary, provide adequate psychotherapy or drug therapy.

\section{Conclusions}

The differences between men and women in the course of ischemic heart disease often are already visible at the level of symptoms, which, in the case of acute coronary syndrome in women, occur less frequently and are often atypical, manifesting in the form of nausea, loss of appetite, or pain of the jaw and neck. Chest pain in women also appears later after the onset of ischemia. Therefore, in the case of acute coronary syndrome, women should be subject to special medical care, and the interpretation of diagnostic tests should take into account the differences between the sexes. In contrast to acute coronary syndromes, in the course of stable coronary heart disease, women more often experience chest pain and have a poorer quality of life, as compared to men.

The differences in effects in women and men are also evident in the diagnostic process, and various pathological mechanisms of myocardial ischemia in men and women affect the differences in the results of diagnostic tests. Such a realization is particularly important in the case of coronary angiography, where it should be remembered that the absence of significant coronary artery disease in symptomatic women does not mean that there was no myocardial ischemia. The reason for this is microvascular dysfunction. This is also responsible for the phenomenon of mental stress in- 
duced myocardial ischemia, where under the influence of emotional stress, the symptoms of angina are seen in patients with stable coronary artery disease.

Vasomotor dysfunction of the coronary arteries and their greater susceptibility to constriction under the influence of catecholamines is also the basis for other adverse events associated with ischemic heart disease. Vasomotor dysfunction is particularly frequent in woman, as their coronary vessels are more sensitive to the catecholamines released during mental stress, resulting in spasm and ischemic myocardium.

All these mentioned factors translate into higher mortality and poorer quality of life of women suffering from ischemic heart disease. Therefore, it is so important to provide better psychological support for women with stable CAD, to adapt the diagnostic process to the sex of the patient, to provide more effective treatment and to ensure cardiac rehabilitation in all women with IHD.

\section{Disclosure}

The authors report no conflict of interest.

\section{References}

1. Mendis S. Global Status Report on noncommunicable diseases 2014 report. World Health Organization, Geneva, Switzerland 2014.

2. Townsend N, Nichols M, Scarborough P, et al. Cardiovascular disease in Europe: epidemiological update 2015. Eur Heart Journal 2015; 36: 2696-2705.

3. Humphries KH, Pu A, Gao M, et al. Angina with "normal" coronary arteries: sex differences in outcomes. Am Heart J 2008; 155: 375-381.

4. Bucholz EM, Butala NM, Rathore SS, et al. Sex differences in long-term mortality after myocardial infarction: a systematic review. Circulation 2014; 130: 757-767.

5. Canto JG, Rogers WJ, Goldberg RJ, et al. Association of age and sex with myocardial infarction symptom presentation and in-hospital mortality. JAMA 2012; 307: 813-822.

6. Sabbag A, Matetzky S, Porter A, et al. Sex differences in the management and 5-year outcome of young patients ( $<55$ years) with acute coronary syndromes. Am J Med 2017; 130: 1324.e15-1324.e22.

7. Cenko E, Yoon J, Kedev S, et al. Sex differences in outcomes after STEMI: effect modification by treatment strategy and age. JAMA Intern Med 2018; 178: 632-639.

8. Araújo C, Laszczyńska O, Viana M, et al. Sex differences in presenting symptoms of acute coronary syndrome: the EPIHeart cohort study. BMJ Open 2018; 2: e018798.

9. Sivarajan Froelicher ES, Miller NH, Christopherson D, et al. High rates of sustained smoking cessation in women hospitalized with cardiovascular disease: the Women's Initiative for Nonsmoking (WINS). Circulation 2004; 109: 587-593.

10. Mason JC, Libby P. Cardiovascular disease in patients with chronic inflammation: mechanisms underlying premature cardiovascular events in rheumatologic conditions. Eur Heart J 2015; 36: 482c-489c.

11. Lubiszewska B, Kruk M, Broda G, et al. The impact of early menopause on risk of coronary artery disease (PREmature Coronary Artery Disease In Women-PRECADIW case-control study). Eur J Prev Cardiol 2012; 19 95-101.

12. Eaker ED, Chesebro JH, Sacks FM, et al. Cardiovascular disease in women. Circulation 1999; 88: 1999-2009.

13. Carr MC. The emergence of the metabolic syndrome with menopause. J Clin Endocrinol Metab 2008; 8: 2404-2411.

14. Mudali S, Dobs AS, Ding J, et al. Atherosclerosis risk in communities study. Endogenous postmenopausal hormones and serum lipids: the atherosclerosis risk in communities study. J Clin Endocrinol Metab 2005; 90: 1202-1209.

15. Sederholm Lawesson S, Isaksson RM, Thylén I, et al. Gender differences in symptom presentation of ST-elevation myocardial infarction: an observational multicenter survey study. Int J Cardiol 2018; 264: 7-11.

16. Canto JG, Goldberg RJ, Hand MM, et al. Symptom presentation of women with acute coronary syndromes: myth vs reality. Arch Intern Med 2007; 167: 2405-2413.

17. Khan NA, Daskalopoulou SS, Karp I, et al. GENESIS PRAXY Team. Sex differences in acute coronary syndrome symptom presentation in young patients. JAMA Intern Med 2013; 173: 1863-1871.

18. Mosca L, Barrett-Connor E, Wenger NK. Sex/gender differences in cardiovascular disease prevention: what a difference a decade makes. Circulation 2011; 124: 2145-2154.

19. Mosca L, Benjamin EJ, Berra K, et al. Effectiveness-based guidelines for the prevention of cardiovascular disease in women - 2011 update: a guideline from the American Heart Association. Circulation 2011; 123: $1243-1262$

20. Freisinger E, Sehner S, Malyar NM, et al. Nationwide routine-data analysis of sex differences in outcome of acute myocardial infarction. Clin Cardiol 2018; 41: 1013-1021.

21. Sadowski M, Gasior M, Gierlotka M, et al. Gender-related differences in mortality after ST-segment elevation myocardial infarction: a large multicentre national registry. Eurolntervention 2011; 6: 1068-1072.

22. Pepine CJ, Ferdinand KC, Shaw LJ, et al. ACC CVD in Women Committee. Emergence of nonobstructive coronary artery disease: a woman's problem and need for change in definition on angiography. J Am Coll Cardiol 2015; 66: 1918-1933.

23. Aggarwal NR, Patel NH, Mehta LS, et al. Sex differences in ischemic heart disease: advances, obstacles, and next steps. Circ Cardiovasc Qual Outcomes 2018; 11: e004437.

24. Colella TJ, Gravely S, Marzolini S, et al. Sex bias in referral of women to outpatient cardiac rehabilitation? A meta-analysis. Eur J Prev Cardiol 2015; 22: 423-441.

25. Kwok YS, Kim C, Grady D, et al. Meta-analysis of exercise testing to detect coronary artery disease in women. Am J Cardiol 1999; 83: 660-666.

26. Shaw LJ, Bairey Merz CN, et al. WISE Investigators. Insights from the NHLBI- sponsored Women's Ischemia Syndrome Evaluation (WISE) study: part I: gender differences in traditional and novel risk factors, symptom evaluation, and gender-optimized diagnostic strategies. J Am Coll Cardiol 2006; 47 (Suppl): S4-S20.

27. Gargiulo P, Petretta M, Bruzzese D, et al. Myocardial perfusion scintigraphy and echocardiography for detecting coronary artery disease in hypertensive patients: a meta-analysis. Eur J Nucl Med Mol Imaging 2011; 38: 2040-2049.

28. Cerci MS, Cerci JJ, Cerci RJ, et al. Myocardial perfusion imaging is a strong predictor of death in women. JACC Cardiovasc Imaging 2011; 4: 880888.

29. Min JK, Shaw LJ, Berman DS. The present state of coronary computed tomography angiography: a process in evolution. J Am Coll Cardiol 2010; 55: 957-965.

30. Taylor AJ, Cerqueira M, Hodgson JM, et al. ACCF/SCCT/ACR/AHA/ASE/ ASNC/NASCI/SCAI/SCMR 2010 appropriate use criteria for cardiac computed tomography. A report of the American College of Cardiology Foundation Appropriate Use Criteria Task Force, the Society of Cardiovascular Computed Tomography, the American College of Radiology, the American Heart Association, the American Society of Echocardiography, the American Society of Nuclear Cardiology, the North American Society for Cardiovascular Imaging, the Society for Cardiovascular Angiography and Interventions, and the Society for Cardiovascular Magnetic Resonance. J Am Coll Cardiol 2010; 56: 1864-1894.

31. Smilowitz NR, Sampson BA, Abrecht CR, et al. Women have less severe and extensive coronary atherosclerosis in fatal cases of ischemic heart disease: an autopsy study. Am Heart J 2011; 161: 681-688.

32. Quyyumi AA. Women and ischemic heart disease: pathophysiologic implications from the Women's Ischemia Syndrome Evaluation (WISE) Study and future research steps. J Am Coll Cardiol 2006; 47 (Suppl 3): S66-S71.

33. Jespersen L, Hvelplund A, Abildstrom SZ, et al. Stable angina pectoris with no obstructive coronary artery disease is associated with increased risks of major adverse cardiovascular events. Eur Heart J 2012; 33: 734-744. 
34. Mehta LS, Beckie TM, DeVon HA, et al. American Heart Association Cardiovascular Disease in Women and Special Populations Committee of the Council on Clinical Cardiology, Council on Epidemiology and Prevention, Council on Cardiovascular and Stroke Nursing, and Council on Quality of Care and Outcomes Research. Acute myocardial infarction in women: a scientific statement from the American Heart Association. Circulation 2016; 133: 916-947.

35. Britten MB, Zeiher AM, Schachinger V. Microvascular dysfunction in angiographically normal or mildly diseasedcoronary arteries predicts adverse cardiovascular long-term outcome. Coron Artery Dis 2004; 15 : 259-264.

36. Niccoli G, Burzotta F, Galiuto L, et al. Myocardial no-reflow in humans. J Am Coll Cardiol 2009; 54: 281-292.

37. Aziz A, Hansen HS, Sechtem U, et al. Sex-related differences in vasomo tor function in patients with angina and unobstructed coronary arteries. J Am Coll Cardiol 2017; 70: 2349-2358.

38. Wokhlu A, Pepine CJ. Mental stress and myocardial ischemia: young women at risk. J Am Heart Assoc 2016; 5: pii: e004196.

39. Pimple $\mathrm{P}$, Hammadah $\mathrm{M}$, Wilmot $\mathrm{K}$, et al. Chest pain and mental stress-induced myocardial ischemia: sex differences. Am J Med 2018; 131: 540-547.

40. Hayek SS, Ko YA, Awad M, et al. Depression and chest pain in patients with coronary artery disease. Int J Cardiol 2017; 230: 420-426.

41. Vaccarino V, Sullivan S, Hammadah M, et al. Mental stress-induced-myocardial ischemia in young patients with recent myocardial infarction: sex differences and mechanisms. Circulation 2018; 137: 794-805.

42. Reynolds HR, Hausvater A, Carney K. Test selection for women with suspected stable ischemic heart disease. J Womens Health (Larchmt) 2018; 27: 867-874. 